\title{
Spatially Coupled Codes over the Multiple Access Channel
}

\author{
Shrinivas Kudekar* and Kenta Kasai ${ }^{\dagger}$ \\ * New Mexico Consortium and CNLS, Los Alamos National Laboratory, New Mexico, USA \\ Email: skudekar@lanl.gov \\ $\dagger$ Dept. of Communications and Integrated Systems, Tokyo Institute of Technology, 152-8550 Tokyo, Japan. \\ Email: kenta@comm.ss.titech.ac.jp
}

\begin{abstract}
We consider spatially coupled code ensembles over a multiple access channel. Convolutional LDPC ensembles are one instance of spatially coupled codes. It was shown recently that, for transmission over the binary erasure channel, this coupling of individual code ensembles has the effect of increasing the belief propagation threshold of the coupled ensembles to the maximum a-posteriori threshold of the underlying ensemble. In this sense, spatially coupled codes were shown to be capacity achieving. It was observed, empirically, that these codes are universal in the sense that they achieve performance close to the Shannon threshold for any general binary-input memoryless symmetric channels.

In this work we provide further evidence of the threshold saturation phenomena when transmitting over a class of multiple access channel. We show, by density evolution analysis and EXIT curves, that the belief propagation threshold of the coupled ensembles is very close to the ultimate Shannon limit.
\end{abstract}

\section{INTRODUCTION}

It has long been known that convolutional LDPC (or spatially coupled) ensembles, introduced by Felström and Zigangirov [1], have excellent thresholds when transmitting over general binary-input memoryless symmetric-output (BMS) channels. The fundamental reason underlying this good performance was recently discussed in detail in [2] for the case when transmission takes place over the binary erasure channel (BEC). In the limit of large $L$ and $w$, the spatiallycoupled LDPC code ensemble $(1, r, L, w)$ [2] was shown to achieve the MAP threshold of $(1, r)$ code ensemble (see last paragraph in this section for the definition of the $(1, r, L, w)$ ensemble). This is the reason why they call this phenomena threshold saturation via spatial coupling. In a recent paper [3], Lentmaier and Fettweis independently formulated the same statement as conjecture.

The phenomena of threshold saturation seems not to be restricted to the BEC. By computing EBP GEXIT curves [4], it was observed in [5] that threshold saturation also occurs for general BMS channels. In other words, in the limit of large 1 (keeping $\frac{1}{r}$ constant), $L$ and $w$, the coupled ensemble $(1, r, L, w)$ achieves universally the capacity of the BMS channels under belief propagation (BP) decoding. Such universality is not a characteristic feature of polar codes [6] and the irregular LDPC codes [7]. According to the channel, polar codes need selection of frozen bits [8] and irregular LDPC codes need optimization of degree distributions.
The principle which underlies the good performance of spatially coupled ensembles has been shown to apply to many other problems in communications, and more generally computer science. To mention just a few, the threshold saturation effect (dynamical threshold of the system being equal to the static or condensation threshold) of coupled graphical models has recently been shown to occur for compressed sensing [9], and a variety of graphical models in statistical physics and computer science like the random $K$-SAT problem, random graph coloring, and the Curie-Weiss model [10]. Other communication scenarios where the spatially coupled codes have found immediate application is to achieve the whole rateequivocation region of the BEC wiretap channel [11], and to achieve the symmetric information rate for a class of channels with memory [12].

It is tempting to conjecture that the same phenomenon occurs for transmission over general multi-user channels. We provide some empirical evidence via density evolution (DE) analysis that this is indeed the case. In particular, we compute EXIT curves for transmission over a multiple access channel (MAC) with erasures. We show that these curves behave in an identical fashion to the curves when transmitting over the BEC. We compute fixed points (FPs) of the coupled DE and show that these FPs have properties identical to the BEC case.

For a review on the literature on convolutional LDPC ensembles, we refer the reader to [2] and the references therein. As discussed in [2], there are many basic variants of coupled ensembles. For the sake of convenience of the reader, we quickly review the ensemble $(1, r, L, w)$. This is the ensemble we use throughout the paper as it is the simplest to analyze.

\section{A. (1, r, L, w) Ensemble [2]}

We assume that the variable nodes are at sections $[-L, L]$, $L \in \mathbb{N}$. At each section there are $M$ variable nodes, $M \in$ $\mathbb{N}$. Conceptually we think of the check nodes to be located at all integer positions from $[-\infty, \infty]$. Only some of these positions actually interact with the variable nodes. At each position there are $\frac{1}{r} M$ check nodes. It remains to describe how the connections are chosen. We assume that each of the 1 connections of a variable node at position $i$ is uniformly and independently chosen from the range $[i, \ldots, i+w-1]$, where $w$ is a "smoothing" parameter. In the same way, we assume 
that each of the $r$ connections of a check node at position $i$ is independently chosen from the range $[i-w+1, \ldots, i]$. The design rate of the ensemble $(1, r, L, w)$, with $w \leq 2 L$, is given by

$$
R(\mathrm{l}, \mathrm{r}, L, w)=\left(1-\frac{\mathrm{l}}{\mathrm{r}}\right)-\frac{\mathrm{l}}{\mathrm{r}} \frac{w+1-2 \sum_{i=0}^{w}\left(\frac{i}{w}\right)^{\mathrm{r}}}{2 L+1} .
$$

A discussion on the above ensemble can be found in [2].

\section{Channel Model, Achievable Rate Region, ITERATIVE DECODING AND FACTOR GRAPH}

\section{A. Binary Adder Channel with Erasures}

We consider the simplest synchronous 2-user multiple access channel, the binary adder channel (BAC) with erasure. More precisely, the inputs to the MAC are binary $X_{1}, X_{2} \in$ $\{0,1\}$. The users take on the values 0,1 with equal probability. The subscripts 1,2 denote the two users. The output $Y \in$ $\{0,1,2, ?\}$ is given by

$$
Y= \begin{cases}Z=X_{1}+X_{2} & \text { with probability } 1-\epsilon \\ ? & \text { with probability } \epsilon,\end{cases}
$$

where $\epsilon$ is the fraction of erasures.

\section{B. Achievable Rate Region}

We assume that the two users do not coordinate their transmission. This implies that the joint input distribution has a product form. Let $R_{1}$ and $R_{2}$ denote the transmission rates of the two users. The achievable rate region is given as follows.

$$
\begin{aligned}
R_{1} & \leq I\left(X_{1} ; Y \mid X_{2}\right), \\
R_{2} & \leq I\left(X_{2} ; Y \mid X_{1}\right), \\
R_{1}+R_{2} & \leq I\left(X_{1}, X_{2} ; Y\right) .
\end{aligned}
$$

The mutual information values above can be computed as

$$
\begin{aligned}
I\left(X_{1} ; Y \mid X_{2}\right) & =I\left(X_{2} ; Y \mid X_{1}\right)=1-\epsilon, \\
I\left(X_{1}, X_{2} ; Y\right) & =\frac{3(1-\epsilon)}{2}, \\
I\left(X_{1} ; Y\right) & =I\left(X_{2} ; Y\right)=\frac{1-\epsilon}{2} .
\end{aligned}
$$

The Shannon limit is defined as the ultimate erasure threshold below which both users can successfully decode using any decoder. Thus, the Shannon threshold is given by,

$$
\epsilon_{\mathrm{Sh}}=\min \left(1-R_{1}, 1-R_{2}, 1-\frac{2}{3}\left(R_{1}+R_{2}\right)\right) .
$$

\section{Factor Graph and Iterative Decoding}

Figure 1 shows the factor graph representation used in the BP decoder analysis. The channel output is the vector $y$ and the user inputs are $\underline{x}_{1}$ and $\underline{x}_{2}$. Each user has its own code and there is a function node which connects the two factor graphs (dark squares in Figure 11). This function node represents the channel factor node $p\left(y_{i} \mid x_{1, i}, x_{2, i}\right)$ and we call it the MAC function node (see [13], [14] for details). Figure 11 shows the spatially coupled ensemble used by each user. For the ease of illustration, we show the protograph-based variant of spatially coupled codes. If we do not use coupled codes for

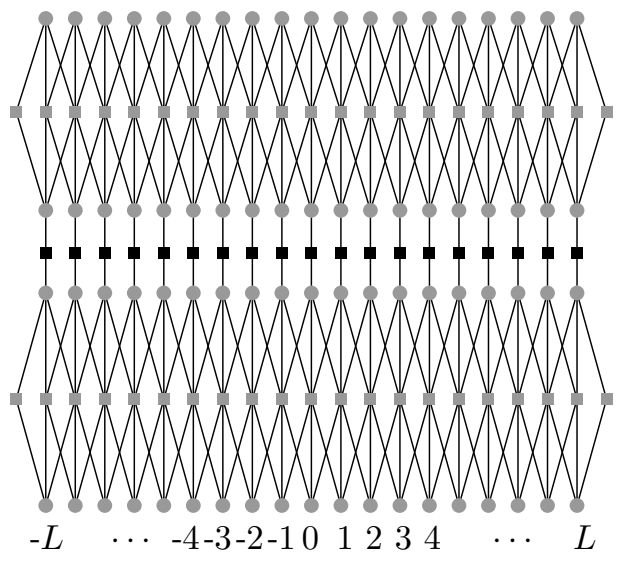

Fig. 1. The figure shows two protograph-based spatially coupled codes (each belonging to one user) in light gray. The two protographs are connected by the MAC function node shown in dark. Note that in the actual code the MAC function node connects each variable node of one user to the corresponding variable node of the other user. For the ease of illustration, we just show connections across one-half of the variable nodes.

transmission, then the two protographs above will be replaced by the usual LDPC codes.

The BP decoder passes messages between the various nodes in the factor graph. The message passing schedule involves first passing the channel observations from the MAC function nodes to the variable nodes of both of the users, then performing one round of BP for both the users (in parallel) and then sending the extrinsic information back to the MAC function node (from both the users).

\section{UnCoupled System: Density Evolution, EXIT-LIKE CURVES}

\section{A. Density Evolution}

Before we proceed to the analysis of coupled codes, it is instructive to consider the $\mathrm{DE}$ analysis for the uncoupled $(1, r)$-regular ensemble. More precisely, users 1 and 2 pick a code from the ensemble $\left(l_{1}, r_{1}\right)$-regular and $\left(l_{2}, r_{2}\right)$-regular respectively. From the schedule given above it is not hard to see that for finite number of iterations and large blocklengths, the local neighborhood around any node is a tree with high probability. See [13], [14] for more details on the DE setup. Also, the BAC with erasures can be thought of as a BEC (for either user) with erasure probability equal to $\epsilon+(1-\epsilon) \mu / 2$, where $\mu$ is the erasure message flowing into the MAC function node. Indeed, the channel output is either erased (wp $\epsilon$ ) or it is not erased (wp $1-\epsilon$ ) and we are still uncertain of the transmitted symbol if the output is equal to 1 (occurs wp 1/2) and the other symbol is uncertain (wp $\mu$ ). The FPs of the DE are then given by,

$$
\begin{aligned}
& y_{1}=1-\left(1-x_{1}\right)^{\mathbf{r}_{1}-1}, \\
& x_{1}=\left(\epsilon+\frac{1-\epsilon}{2} y_{2}^{1_{2}}\right) y_{1}^{1_{1}-1}, \\
& y_{2}=1-\left(1-x_{2}\right)^{\mathbf{r}_{2}-1}, \\
& x_{2}=\left(\epsilon+\frac{1-\epsilon}{2} y_{1}^{1_{1}}\right) y_{2}^{1_{2}-1},
\end{aligned}
$$


where $x_{1}\left(y_{1}\right)$ and $x_{2}\left(y_{2}\right)$ are variable-to-check (check-tovariable) erasure messages of user 1 and 2 respectively. Note that if $l_{1}=l_{2}=1$ and $r_{1}=r_{2}=r$, then the above equations reduce to a single parameter equation and is given by

$$
\begin{aligned}
& x=\left(\epsilon+\frac{(1-\epsilon)}{2} y^{1}\right) y^{1-1}, \\
& y=1-(1-x)^{r-1} .
\end{aligned}
$$

\section{B. Exit-like Curves}

We define the BP EXIT-like 1 function as follows.

$$
h^{\mathrm{BP}}(\epsilon)=\frac{3}{2} y_{1}^{\mathrm{I}_{1}} y_{2}^{\mathrm{I}_{2}}+y_{1}^{\mathrm{I}_{1}}\left(1-y_{2}^{\mathrm{I}_{2}}\right)+\left(1-y_{1}^{\mathrm{I}_{1}}\right) y_{2}^{\mathrm{I}_{2}} .
$$

An intuitive reason as to why we define the BP EXIT function as above is since the entropy of $Z_{i}=X_{1, i}+X_{2, i}$ is $H(1 / 4,1 / 4,1 / 2)=3 / 2$ when a priori messages from both LDPC codes are erased and since the entropy of $Z_{i}$ is 1 when either of them is erased and the other is not.

Assume that all the FPs are parametrized with $x_{1}$ such as $\left(x_{1}, y_{1}\left(x_{1}\right), x_{2}\left(x_{1}\right), y_{2}\left(x_{1}\right), \epsilon\left(x_{1}\right)\right)$. This assumption is true if $\left(\mathrm{l}_{1}, \mathrm{r}_{1}\right)=\left(\mathrm{l}_{2}, \mathrm{r}_{2}\right)=(\mathrm{l}, \mathrm{r})$ with

$$
\begin{aligned}
y_{1}\left(x_{1}\right) & =y_{2}\left(x_{1}\right)=1-\left(1-x_{1}\right)^{\mathrm{r}-1}, \\
x_{2}\left(x_{1}\right) & =x_{1}, \\
\epsilon\left(x_{1}\right) & =\frac{\frac{x_{1}}{y_{1}\left(x_{1}\right)^{1-1}}-\frac{y_{2}\left(x_{1}\right)^{1}}{2}}{1-\frac{y_{2}\left(x_{1}\right)^{1}}{2}} .
\end{aligned}
$$

We then have BP EXIT function as follows

$$
\begin{aligned}
h^{\mathrm{BP}}\left(x_{1}\right)= & \frac{3}{2} y_{1}\left(x_{1}\right)^{1_{1}} y_{2}\left(x_{1}\right)^{1_{2}} \\
& +y_{1}\left(x_{1}\right)^{1_{1}}\left(1-y_{2}\left(x_{1}\right)^{1_{2}}\right) \\
& +\left(1-y_{1}\left(x_{1}\right)^{1_{1}}\right) y_{2}\left(x_{1}\right)^{1_{2}} .
\end{aligned}
$$

We also consider the extended BP EXIT (EBP EXIT) curve which is the plot of all the fixed points of DE. In the case of codes (of each user) being picked from the same ensemble, the EBP EXIT-like curve is given by the parametric curve $\left(h^{\mathrm{BP}}(x), \epsilon(x)\right)$, where $x$ is the variable-to-check node message of either code.

Example 1: Figure 2 shows the plot of the EBP EXIT-like curve for $l_{1}=l_{2}=3, r_{1}=r_{2}=6$. We choose this particular example since as seen from above it is easier to evaluate the value of $\epsilon$ given a fixed value of $x$ (the variable-to-check node message in either of the code). The BP threshold is $\approx 0.12256$ which is much less than the Shannon threshold of $1 / 3$. We observe that if we increase the degrees to $(4,8)$ for both the codes, the BP threshold dramatically drops to zero. Also note the $\mathrm{C}$ shape of the EXIT curve, indicating that there are exactly 3 FPs including a trivial FP plotted at $(0, \epsilon)$ for each channel value, similar to the $\mathrm{BEC}$ case.

\footnotetext{
${ }^{1}$ The reason we call this function EXIT-like is because we do not provide any operational interpretation of these curves like the Area theorem [13]. The curves are drawn only to illustrate that the BP performance of coupled codes is close to the Shannon threshold, which is the main result of the paper.
}

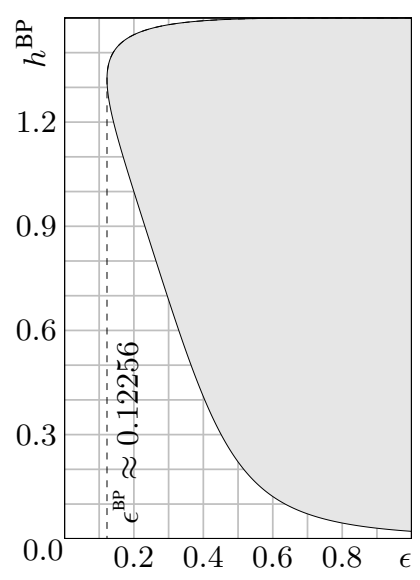

Fig. 2. EBP EXIT curve for the case when both users pick a code from the $(3,6)$ and $(3,6)$. The BP threshold is $\approx 0.12256$ and the Shannon threshold is $1 / 3$.

\section{Main Results}

In this section, we analyze the performance of coupled codes over BAC with erasures. We use the $\left(l_{1}, r_{1}, L, w\right)$ coupled ensemble for user 1 and $\left(1_{2}, r_{2}, L, w\right)$ ensemble for user 2. As a shorthand notation we use $\left(l_{1}, r_{1}, l_{2}, r_{2}, L, w\right)$ to denote both the ensembles. Our main result is that, via DE analysis, the BP threshold of the coupled ensemble is very close to the Shannon threshold given by (1). Furthermore, by increasing the degrees, the BP threshold of the coupled ensemble goes to the Shannon threshold.

Next, we develop the DE equation when transmitting using the coupled codes.

\section{A. Density Evolution for the $\left(\mathrm{l}_{1}, \mathrm{r}_{1}, \mathrm{l}_{2}, \mathrm{r}_{2}, L, w\right)$ ensemble}

We develop the DE equations assuming that the two users use ensembles of different degrees. Consider the $\left(\mathrm{l}_{1}, \mathrm{r}_{1}, \mathrm{l}_{2}, \mathrm{r}_{2}, L, w\right)$ ensemble. To perform the DE analysis, we already take the limit $M \rightarrow \infty$ (the number of variable nodes in each section).

Let $x_{1, i}, i \in \mathbb{Z}$, denote the average erasure probability which is emitted by variable nodes at position $i$ to check nodes at position $i$ for user 1 . Similarly define $x_{2, i}$ for the user 2 . For $i \notin[-L, L]$, we set $x_{1, i}=x_{2, i}=0$. For $i \in[-L, L]$ the DE is given by

$$
\begin{aligned}
& y_{1, i}=1-\left(1-\frac{1}{w} \sum_{k=0}^{w-1} x_{1, i-k}\right)^{\mathrm{r}_{1}-1}, \\
& x_{1, i}=\left(\epsilon+\frac{1-\epsilon}{2}\left(\frac{1}{w} \sum_{j=0}^{w-1} y_{2, i+j}\right)^{1_{2}}\right)\left(\frac{1}{w} \sum_{j=0}^{w-1} y_{1, i+j}\right)^{\mathrm{l}_{1}-1}, \\
& y_{2, i}=1-\left(1-\frac{1}{w} \sum_{k=0}^{w-1} x_{2, i-k}\right)^{\mathrm{r}_{2}-1}, \\
& x_{2, i}=\left(\epsilon+\frac{1-\epsilon}{2}\left(\frac{1}{w} \sum_{j=0}^{w-1} y_{1, i+j}\right)^{\mathrm{I}_{1}}\right)\left(\frac{1}{w} \sum_{j=0}^{w-1} y_{2, i+j}\right)^{\mathrm{I}_{2}-1} .
\end{aligned}
$$


We will use the notation $\epsilon^{\mathrm{BP}}\left(1_{1}, r_{1}, 1_{2}, r_{2}, L, w\right)$ to denote the threshold of the BP decoder when we use coupled codes for transmission. Also, we use $\epsilon^{\mathrm{BP}}\left(\mathrm{l}_{1}, \mathrm{r}_{1}, \mathrm{l}_{2}, \mathrm{r}_{2}\right)$ to denote the BP threshold of the underlying uncoupled ensemble.

As a shorthand we use $g_{1}\left(x_{i-w+1}^{1,2}, \ldots, x_{i+w-1}^{1,2}\right)$ to denote $\left(\epsilon+\frac{1-\epsilon}{2}\left(\frac{1}{w} \sum_{j=0}^{w-1} y_{2, i+j}\right)^{1_{2}}\right)\left(\frac{1}{w} \sum_{j=0}^{w-1} y_{1, i+j}\right)^{1_{1}-1}$ and also $g_{2}\left(x_{i-w+1}^{1,2}, \ldots, x_{i+w-1}^{1,2}\right)$ to denote $(\epsilon+$ $\left.\frac{1-\epsilon}{2}\left(\frac{1}{w} \sum_{j=0}^{w-1} y_{1, i+j}\right)^{1_{1}}\right)\left(\frac{1}{w} \sum_{j=0}^{w-1} y_{2, i+j}\right)^{1_{2}-1}$.

Definition 2 (FPs of Density Evolution): Consider $\mathrm{DE}$ for the $\left(\mathrm{I}_{1}, \mathrm{r}_{1}, \mathrm{I}_{2}, \mathrm{r}_{2}, L, w\right)$ ensemble. Let $\underline{x}_{1}=\left(x_{1,-L}, \ldots, x_{1, L}\right)$ and $\underline{x}_{2}=\left(x_{2,-L}, \ldots, x_{2, L}\right)$ denote the vector of variable-to-check erasure messages for user 1 and 2 respectively. We call $\underline{x}_{1}$ and $\underline{x}_{2}$ the constellation of user 1 and 2 respectively. We say that $\left(\underline{x}_{1}, \underline{x}_{2}\right)$ forms a FP of DE with channel $\epsilon$ if $\left(\underline{x}_{1}, \underline{x}_{2}\right)$ fulfills 3 for $i \in[-L, L]$. As a shorthand we then say that $\left(\epsilon, \underline{x}_{1}, \underline{x}_{2}\right)$ is a FP. We say that $\left(\epsilon, \underline{x}_{1}, \underline{x}_{2}\right)$ is a non-trivial FP if either $\underline{x}_{1}$ or $\underline{x}_{2}$ is not identically equal to $0 \forall i$. Again, for $i \notin[-L, L]$, $x_{1, i}=x_{2, i}=0$.

Definition 3 (Forward DE and Admissible Schedules):

Consider forward $\mathrm{DE}$ for the $\left(\mathrm{l}_{1}, \mathrm{r}_{1}, \mathrm{l}_{2}, \mathrm{r}_{2}, L, w\right)$ ensemble. More precisely, pick a channel $\epsilon$ and initialize $\underline{x}_{1}^{(0)}=\underline{x}_{2}^{(0)}=(1, \ldots, 1)$. Let $\underline{x}_{1}^{(\ell)}$ and $\underline{x}_{2}^{(\ell)}$ be the result of $\ell$ rounds of DE for user 1 and 2 respectively. More precisely, $\underline{x}_{1}^{(\ell+1)}$ and $\underline{x}_{2}^{(\ell+1)}$ are generated from $\underline{x}_{1}^{(\ell)}$ and $\underline{x}_{2}^{(\ell)}$ by applying the DE equation (3) to each section $i \in[-L, L]$,

$$
\begin{aligned}
& x_{1, i}^{(\ell+1)}=g_{1}\left(x_{i-w+1}^{1,2,(\ell)}, \ldots, x_{i+w-1}^{1,2,(\ell)}\right), \\
& x_{2, i}^{(\ell+1)}=g_{2}\left(x_{i-w+1}^{1,2,(\ell)}, \ldots, x_{i+w-1}^{1,2,(\ell)}\right),
\end{aligned}
$$

where we use the notation $x_{i}^{1,2,(\ell)}$ to denote $\left(x_{1, i}^{(\ell)}, x_{2, i}^{(\ell)}\right)$. We call this the parallel schedule.

More generally, consider a schedule in which in each step $\ell$ an arbitrary subset of the sections is updated, constrained only by the fact that every section is updated in infinitely many steps. We call such a schedule admissible. Again, we call $\underline{x}_{1}^{(\ell)}$ and $\underline{x}_{2}^{(\ell)}$ the resulting sequence of constellations.

One can show that if we perform forward DE under any admissible schedule, then the constellations $\underline{x}_{1}^{(\ell)}$ and $\underline{x}_{2}^{(\ell)}$ converge to a FP of DE and this FP is independent of schedule. This statement can be proved similar to the one in [2], [13].

For the case when $l_{1}=l_{2}$ and $r_{1}=r_{2}$ we have that for any FP, $x_{1, i}=x_{2, i}$ and $y_{1, i}=y_{2, i}$ for all $i$.

\section{B. Forward DE - Simulation Results}

In the examples below, the Shannon threshold is computed using equation (1).

Example 4 (Equal Degrees - BP goes to Shannon):

We consider forward $\mathrm{DE}$ for the coupled ensembles. More precisely, we fix an $\epsilon$ and initialize all $x_{1, i}$ and $x_{2, i}$ to 1 , for $i \in[-L, L]$. Then we run the $\mathrm{DE}$ given by (3) till we reach a fixed-point. We fix $L=200$. For $l_{1}=l_{2}=3$ and $r_{1}=r_{2}=6$, we have that $\epsilon^{\mathrm{BP}}(3,6,3,6,200,3) \approx 0.332287$. If we increase the degrees we get $\epsilon^{\mathrm{BP}}(4,8,4,8,200,4) \approx 0.333195$, $\epsilon^{\mathrm{BP}}(5,10,5,10,200,5) \approx 0.333286$. We observe that by increasing the degrees the BP threshold approaches the
Shannon threshold of $1 / 3$. On the other hand for the uncoupled codes, $\epsilon^{\mathrm{BP}}(3,6,3,6) \approx 0.12256$ and for larger degrees the BP threshold is zero.

Example 5 (Unequal Degrees - BP goes to Shannon): We also consider the more general case when the degrees are not equal. For $l_{1}=5, r_{1}=10$ and $l_{2}=6, r_{2}=13$ we get $\epsilon^{\mathrm{BP}}(5,10,6,13,500,10) \approx 0.307647$. The Shannon threshold in this case is equal to $\approx 0.307692$. For $1_{1}=9, r_{1}=10$ and $\mathrm{I}_{2}=6, \mathrm{r}_{2}=10$ we get $\epsilon^{\mathrm{BP}}(9,10,6,10,500,10) \approx 0.59992$ and the Shannon threshold is $=0.6$.

\section{EXIT curve plots}

We also show via EXIT analysis that the coupling of regular LDPC codes pushes the BP threshold (of the coupled systems) to the Shannon threshold. For the purpose of illustration of the threshold saturation phenomena we focus only on the case when $l_{1}=l_{2}$ and $r_{1}=r_{2}$. Thus, the variable-to-check node messages, for any FP of DE, for both the users are equal (cf. (3)). Now, to plot the EBP EXIT curve, which is essentially the plot of all the fixed-points of DE, we define the entropy of a constellation as

$$
\chi=\frac{1}{2 L+1} \sum_{i=-L}^{L} x_{1, i} .
$$

To plot all the FPs of DE, we first fix a value of $\chi \in[0,1]$ and then run the reverse DE process given in [4]. Briefly, we start with an initial variable-to-check message and run it through the check node. Then the appropriate channel value is found such that the resulting constellation has entropy equal to $\chi$. This process is run till we get an FP. Figure 3 shows the plot of the EBP EXIT curve for the $(3,6,3,6, L, 3)$ ensemble with $L=2,4,8,16,32,64,128,256$. We observe that the plot looks very similar to the case of single user transmission over a BEC. For small values of $L$ there is a large rateloss and the EBP EXIT curve is to the right. As $L$ increases, the rateloss diminishes and the curves move to the left. The limiting BP EXIT curve of the coupled system looks very similar to when we are transmitting over the BEC. It traces the BP EXIT function of the underlying uncoupled codes until the channel erasure value is very close to the Shannon threshold and then drops vertically to almost zero entropy.

\section{Shape of the Constellation}

Figure 4 shows the constellation of an unstable FP (which cannot be reached by BP). This FP is obtained via the reverse DE process. This special FP was the key ingredient in proving threshold saturation over the BEC [2]. Let us describe the (empirically observed) crucial properties of this constellation.

(i) The constellation is symmetric around $i=0$ and is unimodal. The constellation has $\epsilon \approx 0.3323$, which is close to the Shannon threshold of $1 / 3$.

(ii) The value in the flat part in the middle is $\approx 0.6548$ which is very close to the stable FP of DE for the underlying uncoupled $(3,6)$-regular ensemble at $\epsilon \approx 0.3323$.

(iii) The transition from values close to zero to values close to 0.6548 is very quick. 


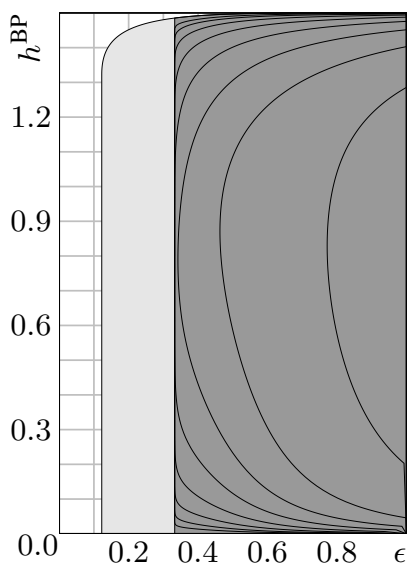

Fig. 3. The EBP EXIT curve for $(3,6,3,6, L, 3)$ with $L=$ $2,4,8,16,32,64,128,256$. The curve with light gray background is the BP EXIT curve for the uncoupled $(3,6,3,6)$ ensemble. We see that as $L$ increases the EBP EXIT curves of the coupled system moves to the left. The BP threshold of the coupled system is $\approx 0.3323$ which is very close to the Shannon threshold.

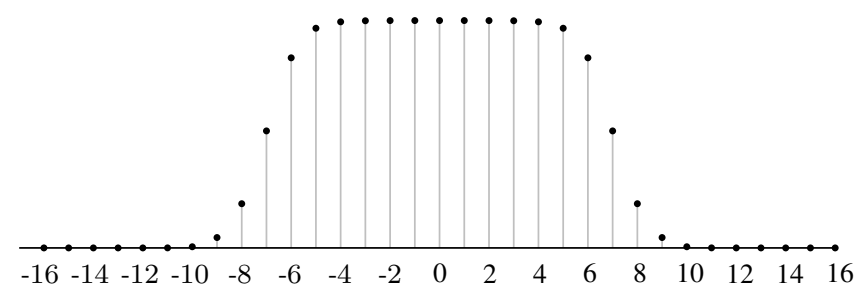

Fig. 4. The unstable FP shown above has an entropy of 0.28 and is obtained via reverse DE. The constellation is symmetric around 0 and is unimodal. The flat middle part has value close to 0.6548 which is the value of stable FP for the uncoupled system at $\epsilon \approx 0.3323$. Both the users have identical FP constellation.

\section{Discussion}

In this paper we show that, by using coupled regular LDPC codes when transmitting over the 2 user BAC with erasures, the BP threshold can be made very close to the Shannon threshold. In this sense, the coupled codes are threshold saturating. We demonstrate this by plotting EXIT-like curves. The behavior of these curves is very similar to when transmitting over the BEC. Even the shape of the constellation of an unstable FP of DE is same as the BEC case. Thus we believe one should be able to provide a proof of this phenomena on the lines of the BEC proof [2].

Another interesting question is to determine area theorems which will also further show that the BP threshold of the coupled system goes to the MAP threshold of the underlying uncoupled codes, when we consider finite degrees. To do this we would need to define an appropriate EXIT function.

Lastly, it would be interesting to see if we can demonstrate the threshold saturation phenomena to more general MAC channels, like the 2 user BAC with additive Gaussian noise.

\section{ACKNOWLEDGMENTS}

SK acknowledges support of NMC via the NSF collaborative grant CCF-0829945 on "Harnessing Statistical Physics for Computing and Communications." SK would also like to thank Rüdiger Urbanke for his encouragement.

\section{REFERENCES}

[1] A. J. Felström and K. S. Zigangirov, "Time-varying periodic convolutional codes with low-density parity-check matrix," IEEE Trans. Inform. Theory, vol. 45, no. 5, pp. 2181-2190, Sept. 1999.

[2] S. Kudekar, T. Richardson, and R. Urbanke, "Threshold saturation via spatial coupling: Why convolutional LDPC ensembles perform so well over the BEC," 2010, e-print: http://arxiv.org/abs/1001.1826.

[3] M. Lentmaier and G. P. Fettweis, "On the thresholds of generalized LDPC convolutional codes based on protographs," in Proc. of the IEEE Int. Symposium on Inform. Theory, Austing, TX, USA, June 2010, pp. 709-713.

[4] C. Méasson, A. Montanari, T. Richardson, and R. Urbanke, "The generalized area theorem and some of its consequences," IEEE Trans. Inform. Theory, vol. 55, no. 11, pp. 4793-4821, Nov. 2009.

[5] S. Kudekar, C. Méasson, T. Richardson, and R. Urbanke, "Threshold saturation on BMS channels via spatial coupling," Apr. 2010, e-print: http://arxiv.org/abs/1004.3742.

[6] E. Arıkan, "Channel polarization: A method for constructing capacityachieving codes for symmetric binary-input memoryless channels," IEEE Trans. Inform. Theory, vol. 55, no. 7, pp. 3051-3073, 2009.

[7] T. Richardson, A. Shokrollahi, and R. Urbanke, "Design of capacityapproaching irregular low-density parity-check codes," IEEE Trans. Inform. Theory, vol. 47, no. 2, pp. 619-637, Feb. 2001.

[8] R. Mori and T. Tanaka, "Performance and construction of polar codes on symmetric binary-input memoryless channels," Jan. 2009, http://arxiv.org/abs/0901.2207.

[9] S. Kudekar and H. D. Pfister, "The effect of spatial coupling on compressive sensing," in Proc. of the Allerton Conf. on Commun., Control, and Computing, Monticello, IL, USA, 2010.

[10] S. H. Hassani, N. Macris, and R. Urbanke, "Coupled graphical models and their thresholds," in Proc. of the IEEE Inform. Theory Workshop, Dublin, Ireland, Sept. 2010.

[11] V. Rathi, R. Urbanke, M. Andersson, and M. Skoglund, "Rateequivocation optimally spatially coupled LDPC codes for the BEC wiretap channel," 2010, e-print: http://arxiv.org/abs/1010.1669.

[12] S. Kudekar and K. Kasai, "Threshold Saturation on Channels with Memory via Spatial Coupling,” 2011, e-print: http://arxiv.org/abs/0211.1669.

[13] T. Richardson and R. Urbanke, Modern Coding Theory. Cambridge University Press, 2008.

[14] A. Amraoui, S. Dusad, and R. Urbanke, "Achieving general points in the 2-user Gaussian MAC without time-sharing or rate-splitting by means of iterative coding," in Proc. of the IEEE Int. Symposium on Inform. Theory, Lausanne, Switzerland, June 2002, conference, p. 334. 